Tropical Journal of Pharmaceutical Research February 2013; 12 (1): 7-11

ISSN: $1596-5996$ (print); 1596-9827 (electronic)

(c) Pharmacotherapy Group, Faculty of Pharmacy, University of Benin, Benin City, 300001 Nigeria.

All rights reserved.

Available online at http://www.tjpr.org

Original Research Article

http://dx.doi.org/10.4314/tjpr.v12i1.2

\title{
Green Synthesis of Silver Nanoparticles Induced by the Fungus Penicillium citrinum
}

\author{
Soheyla Honary ${ }^{1^{*}}$, Hamed Barabadi ${ }^{1}$, Eshrat Gharaei-Fathabad ${ }^{1}$ and Farzaneh \\ Naghibi $^{2}$ \\ ${ }^{1}$ Mazandaran University of Medical Sciences, School of pharmacy, Sari, and ${ }^{2}$ School of Traditional Medicine, Shaheed \\ Beheshti University of Medical Sciences, Tehran, Iran.
}

*For correspondence: Email: shonary@mazums.ac.ir, shonary@yahoo.com; Tel: 00989121452220

Received: 5 April 2012

Revised accepted: 4 December 2012

\begin{abstract}
Purpose: To evaluate a green process for the extracellular production of silver ( $\mathrm{Ag}$ ) nanoparticles synthesized and stabilized using Penicillium citrinum isolated from soil.

Methods: The pure colonies of Penicillium citrinum were cultured in Czapek dox broth. The supernatant of the broth was examined for the ability to produce silver nanoparticles. The reactions were performed in a dark compartment at $28{ }^{\circ} \mathrm{C}$. After $24 \mathrm{~h}$, the synthesized silver nanoparticles were filtered through a membrane filter $(0.45 \mu)$ and characterized by UV-visible spectroscopy, fluorescence spectroscopy, photon correlation spectroscopy (PCS), scanning electron microscopy (SEM) and Fourier transformed infrared spectroscopy (FTIR) for particle size, shape and the presence of different functional groups in the nanoparticles.

Results: The silver nanoparticles formed were fairly uniform in size with a spherical shape and a Zaverage diameter of $109 \mathrm{~nm}$. FTIR spectra revealed the presence of amide linkage groups which were also found in the fungal extract itself.

Conclusion: The current approach suggests that rapid synthesis of nanoparticles of silver nitrate would be suitable for developing a biological process for mass scale production of formulations.
\end{abstract}

Keywords: Green synthesis, Penicillium citrinum, silver nanoparticles.

Tropical Journal of Pharmaceutical Research is indexed by Science Citation Index (SciSearch), Scopus, International Pharmaceutical Abstract, Chemical Abstracts, Embase, Index Copernicus, EBSCO, African Index Medicus, JournalSeek, Journal Citation Reports/Science Edition, Directory of Open Access Journals (DOAJ), African Journal Online, Bioline International, Open-J-Gate and Pharmacy Abstracts

\section{INTRODUCTION}

Nanotechnology has recently become one of the most active research fields in technology and engineering. Nanotechnology possesses a potential impact on energy consumption [1] and can also aid in solving major health problems due to the production more effective drugs [1]. Although the products of this technology will be cheaper, they promise more functionality and require less energy and fewer raw materials to manufacture [1-2].
The synthesis of metal and semiconductors nanoparticles is important because of their novel electrical, optical, magnetic, and chemical properties. Several methods have been used to prepare metal nanoparticles, including chemical reduction, photochemical or radiation-chemical reduction, metallic wire explosion, sonochemical method and polyol [3-6]; however, some of these methods involve the use of toxic chemicals in the synthesis process [7-8]. Therefore, there is an urgent need to develop a 'green' process of nanoparticle synthesis by non-pathogenic microorganisms. 
'Green synthesis' is a process of synthesis and assembly of nanoparticles and has been used for a series of special production processes. This process, benefits from the development of clean, non-toxic and environmentally acceptable procedures which involve organisms ranging from bacteria to fungi and even plants [9]. Fungi have significantly higher productivity when used in nanoparticles biosynthesis owing to their much higher protein secretion [10].

A novel biological method for the intra- and extracellular synthesis of silver nanoparticles by the fungi, Verticillium and Fusarium oxysporum, respectively, has been documented [10-12]. Nanda et al has shown that silver nanoparticles are an effective antimicrobial agent against various pathogenic microorganisms [13]. While a large number of microbial species are capable of producing metal nanoparticles (NPs), the mechanism of nanoparticle biosynthesis is very important. Much remains unknown about the biochemical and molecular mechanisms of these processes remain unknown and therefore there is a need to explore this aspect. The study of the enzyme structure may help improve the size, shape and dispersability of the generated metal nanoparticles.

Although colloidal silver, as an antibacterial agent, was discovered at the beginning of the 20th century, however, growing resistance of microorganisms to antibiotics has brought renewed interest in the biological activity of this form of silver [14]. This study therefore explores an in vitro approach for the biosynthesis of silver nanoparticles using the fungus, Penicillium citrinum.

\section{EXPERIMENTAL}

Yeast extract was purchased from Liofilchem, Italy while silver nitrate and other chemical reagents were purchased from Merck, Germany.

\section{Biosynthesis of silver nanoparticles}

The fungus Penicillium citrinum was isolated from soil and confirmed in the medical biotechnology laboratory, Department of Biotechnology, Mazandaran University of Medical Science, Iran. The microbe was cultured on fluid zapex dox broth, incorporating $21 \mathrm{~g}$ sucrose and $3 \mathrm{~g}$ yeast extract in $1000 \mathrm{ml}$ distilled water, and then incubated at $28^{\circ} \mathrm{C}$ at $200 \mathrm{rpm}$ for seven days (IKA KS 4000). The culture was centrifuged at $10,000 \mathrm{rpm}$ for $5 \mathrm{~min}$, and the supernatant was used for the synthesis of silver nanoparticles. A solution of silver nitrate $(0.1 \mathrm{mM})$ was prepared by dissolving $0.017 \mathrm{~g}$ of the compound in $100 \mathrm{ml}$ of double distilled water. Thereafter, $100 \mathrm{ml}$ of silver nitrate solution at a concentration of $1 \mathrm{mM}$ was added to $100 \mathrm{ml}$ of the supernatant and incubated again for 24 hours at $28^{\circ} \mathrm{C}$ (total concentration of silver nitrate $=0.5$ $\mathrm{mM})$. The colors of the solutions changed to dark buff, indicating the formation of silver nanoparticles in the solution. The solutions were centrifuged at $1,000 \mathrm{~g}$, the particles separated from the supernatant and dried in $40^{\circ} \mathrm{C}$. Finally, the silver nanoparticles were stored carefully in dark vials for further analysis.

\section{Evaluation of nanoparticles}

The reduction of silver nitrate to silver nanoparticles was confirmed by ultraviolet (UV) spectroscopy (Genesys 2 spectrophotometer, USA). The silver nanoparticles were evaluated for their fluorescent characteristics by PerkinElmer LS 50B luminescence spectrophotometer, and for their surface and shape characteristics by scanning electron microscope (model 2360, Leo Oxford, England). The SEM image is capable of providing two main types of data, namely, the topographic structure of the surface and the differentiation of thet phases in the sample. Size and polydispersity of particles were determined by Zetasizer Nano Particle Analyzer (model 3600 , Malvern instruments, UK) at $25^{\circ} \mathrm{C}$ at a scattering angle of $90^{\circ}$. Photon correlation spectroscopy (PCS) is a non-invasive technique that measures the size and size distribution of nanoparticles dispersed in a liquid.

Fourier-transform infrared (FTIR) spectrum was obtained by mixing with potassium bromide at $1: 100$ ratio which was compressed to a $2 \mathrm{~mm}$ semi-transparent disk for $2 \mathrm{~min}$. Spectra over the wavelength range $4000-400 \mathrm{~cm}^{-1}$ were recorded using FTIR spectrometer (Spectrum one, Perkin Elmer, Germany).

\section{RESULTS}

Addition of Penicillium citrinum biomass to $1 \mathrm{mM}$ aqueous $\mathrm{AgNO}_{3}$ solution led to a colour change to yellowish brown in the solution after $24 \mathrm{~h}$ of reaction, indicating the formation of silver nanoparticles. The UV-vis spectrum exhibited an absorption band at around $400-420 \mathrm{~nm}$ which is a typical plasmon band (Fig 1A). Furthermore, the UV-vis spectrum revealed that the reaction medium exhibited an absorption band around $265 \mathrm{~nm}$ which is attributed to aromatic amino acids of proteins. The fluorescence spectrum showed a broad emission peak of silver nanoparticles at $414 \mathrm{~nm}$ when excited at $432 \mathrm{~nm}$ (Fig 1B). 

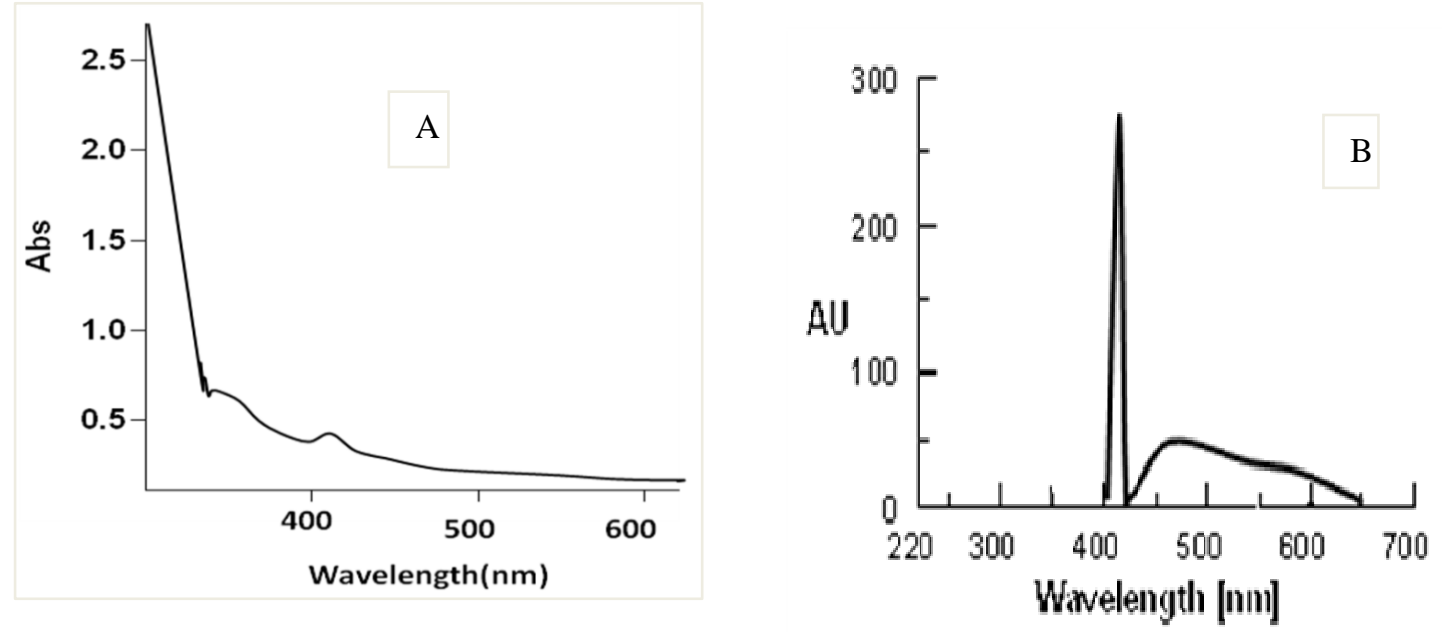

Figure 1: UV-visible absorption (A) and fluorescence spectrum (B) spectrum of the silver nanoparticles

Figure 2 shows that the Z-average size of the silver nanoparticles is $109 \mathrm{~nm}$ with a polydispersity index (PDI) of 0.1 . The results of scanning electron microscopy (SEM) show that the silver nanoparticles have a uniform spherical shape with a size range of $90-120 \mathrm{~nm}$ (Fig 3). The FTIR spectrum of silver nanoparticles indicate that the nanoparticles manifest absorption peaks at about 1053.89, 1412.95 and $1626.37 \mathrm{~cm}^{-1}$ which represent amide linkages groups. Furthermore, the peaks near 3401 and $2919 \mathrm{~cm}^{-1}$ were assigned to $\mathrm{OH}$ stretching. The band at $1626 \mathrm{~cm}^{-1}$ corresponds to amide I due to carbonyl stretch in proteins. The peak at 1041 $\mathrm{cm}^{-1}$ corresponds to $\mathrm{C}-\mathrm{N}$ stretching vibration of amine (fig 4).

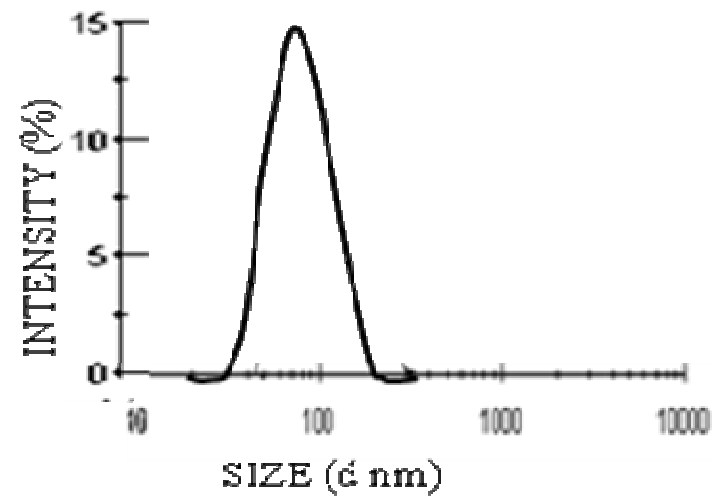

Figure 2: Photon correlation spectroscopy (PCS) of the silver nanoparticles; Z-average size $=109 \mathrm{~nm}$, PDI: 0/1

\section{DISCUSSION}

The color change from pale yellow to yellowish brown when $1 \mathrm{mM}$ silver nitrate was added to the solution is due to the excitation of surface plasmon vibrations in the metal nanoparticles.

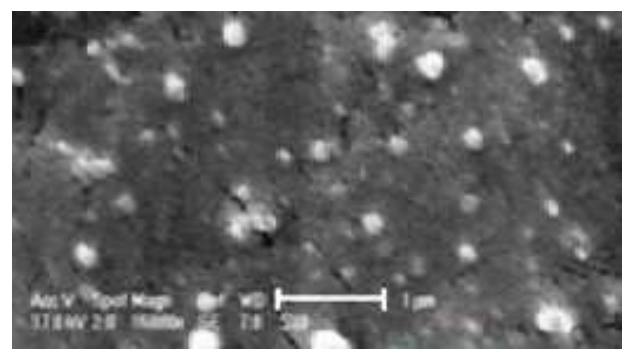

Figure 3: SEM image of the silver nanoparticles

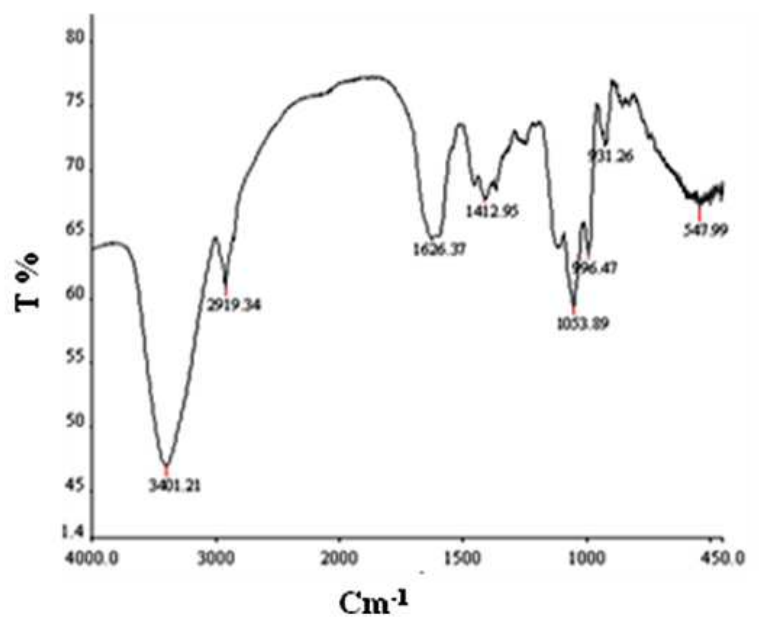

Figure 4: FTIR spectrum of silver nanoparticles synthesized by Penicillium citrinum.

Control without silver ions showed no change in color when incubated under the same conditions. Many metals can be treated as free-electron systems. These metals, called plasma, contain equal numbers of positive ions (which are fixed in position) and conduction electrons (which are free and highly mobile). Under the irradiation of an electromagnetic wave, the free electrons are driven by the electric field to oscillate coherently. These collective oscillations of the free electrons are called plasmons. These plasmons can 
interact, under certain conditions, with visible light in a phenomenon called surface plasmon resonance (SPR) [15-16]. SPR plays a major role in the determination of optical absorption spectra of metal nanoparticles, which shifts to a longer wavelength as the particle size increases [17].

The UV-vis spectrum of silver nanoparticles produced by Penicillium citrinum exhibited an absorption band at around $400-420 \mathrm{~nm}$ which is a typical plasmon band, suggesting the formation of silver nanoparticles. It is has been reported that the absorption band at $265 \mathrm{~nm}$ is due to electronic excitation in tryptophan and tyrosine residues in protein [18-19]. Also, the nature of the fluorescence emission band at $414 \mathrm{~nm}$ indicates that the proteins bound to the nanoparticle surface and those present in the solution exist in the native form [18]. We believe that the presence of proteins in the fungal biomass plays an important role in nanoparticle synthesis and stabilization.

PCS spectra demonstrated that the silver NPs formed had fairly well-defined dimensions and good monodispersity. As a result of the larger surface area and attractive force between the particles, the likelihood of aggregation is high for small-sized particles. PCS facilitates the understanding of the dispersion and aggregation. polydispersity index (PDI) measures the second moment of the size distribution of the nanoparticle population. PDI ranges from a value of 0.01 to $0.5-0.7$ for monodispersed particles. Samples with very broad size distribution have polydispersity index $>0.7$ [20].

FTIR aids in identifying the possible biomolecules responsible for capping and efficient stabilization of the metal nanoparticles synthesized by Penicillium citrinum. The FTIR spectrum revealed the presence of different functional groups such as amide linkages and COO- which are probably sandwiched between amino acid residues in the protein and the synthesized silver nanoparticles.

\section{CONCLUSION}

Compared to other methods, 'green' method seems to be less costly, simpler and yet more functional, and also would require less energy and fewer raw materials for production. This study show that zero valent metal nanoparticles can be obtained by bio-reduction of metal salts. Green method of producing small and uniform silver nanoparticles can be further developed as an alternative method for the production of nanoparticles on a large scale.

\section{ACKNOWLEDGMENT}

This work was financially supported by Traditional Medicine and Materia Medica Research Center (TMRC), Shaheed Beheshti University of Medical Sciences, Tehran, Iran.

\section{REFERENCES}

1. Bae D, Kim E, Bang J, Kim S, Han K, Lee J, Kim B, Adair J. Synthesis and Characterization of Silver Nanoparticles by a Reverse Micelle Process. Met Mater Int 2005; 4: 291-294.

2. Honary $S$, Ghajar $K$, Khazaeli $P$, Shalchian $P$. Preparation, Characterization and Antibacterial Properties of Silver-Chitosan Nanocomposites Using Different Molecular Weight Grades of Chitosan. Trop. J. Pharm. Res. 2011; 10: 69-74.

3. Chou KS, Ren CY. Synthesis of nanosized silver particles by chemical reduction method. Mater Chem Phys 2000; 64: 241-249.

4. Zhou HS, Wada T, Sasabe H, Komiama H. Synthesis of nanometer-size silver coated polymerized diacetylene composite particles. Appl Phys Lett 1996; 68: 1288-1293.

5. Okitsu K, Bandow $H$, Maeda Y. Sono-chemical preparation of ultrafine palladium particles. Chem Mater 1996; 8: 315-317.

6. Silvert PY, Herrera-Urbina $R$, Duvauchellc $N$, Vijayakrishnan V. Preparation of colloidal silver dispersion by th polyol process-partz: mechanism of particle formation. J Mater Chem 1996; 6: 573577.

7. Azim A, Davood Z, Ali F, Mohammad RM, Dariush N, Shahram T, Majid M, Nasim B. Synthesis and characterization of gold nanoparticles by tryptophane. Am J Appl Sci 2009; 6: 691-695.

8. Tapan KS, Andrey LR. Nonspherical noble metal nanoparticles: colloidchemical synthesis and morphology control. Adv Mater 2009; 21: 1-24.

9. Mohanpuria P, Rana NK, Yadav SK. Biosynthesis of nanoparticles: technological concepts and future applications. J Nanopart Res 2008; 10: 507-517.

10. Mukherjee $P$, Ahmad A, Mandal D, Senapati S, Sainkars $S$, Khan M, Parishcha R, Ajavkumar P, Alam M, Kumar R, Sastry M, Fungus-mediated synthesis of silver nanoparticles and their immobilization in the mycelia matrix: a novel biological approach to nanoparticle synthesis. Nano Lett 2001; 1: 515519.

11. Senapati S, Mandal D, Ahmad A, Khan MI, Sastry M, Kumar R. Fungus mediated synthesis of silver nanoparticles: a novel biological approach. Indian J Phys 2004; 78: 101-105.

12. Mandal D, Bolander ME, Mukherjee $P$, Mukhopadhyay $D$, Sarkar $G$. The use of microorganisms for the formation of metal nanoparticles and their application. Appl Microbiol Biotechnol 2006; 69: 485-492

13. Nanda A, Saravanan M. Biosynthesis of silver nanoparticles from Staphylococcus aureus and its antimicrobial activity against MRSA and MRSE. Nanomedicine: NBM 2009; 5: 452-456.

14. Bera T, Ramachandrarao P. Morphological changes in biomimetically synthesized hydroxyapatite and silver nanoparticles for medical applications. J Mater Sci 2009; 44: 2264-2270.

15. Moores A, Goettmann $F$. The plasmon band in noble metal nanoparticles: an introduction to theory and applications. NJC 2006; 30: 1121-1132.

16. Pitark JM, Silkin VM, Chulkov EV, Echenique PM. Surface plasmons in metallic structures. J Optic Pure Appl Optic 2005; 7: 73-84. 
17. Zhao $Y$, Jiang $Y$, Fang $Y$. Spectroscopy property of $A g$ nanoparticles. Acta A 2006; 65: 1003-1006.

18. Durán N, Marcato PD, Alves OL, Souza Gl, Esposito E. Mechanistic aspects of biosynthesis of silver nanoparticles by several Fusarium oxysporum strains. JoN 2005; 3: 8 doi:10.1186/1477-3155-3-8

19. Ahmad A, Mukherjee $P$, Senapati S, Mandal D, Khan MI, Kumar R, Sastry M. Extracellular biosynthesis of silver nanoparticles using the fungus Fusarium oxysporum. Colloids Surf B 2003; 28: 313-318.

20. Nidhin M, Indumathy $R$, Sreeram KJ, Nair BU. Synthesis of iron oxide nanoparticles of narrow size distribution on polysaccharide templates. Bull Mater Sci 2008; 31: 93-96. 